\title{
Bias Robust Channel Prediction for OFDM based Adaptive Modulation
}

\author{
Hoiyoon Jung, Hyoungsuk Jeon, Jongsub, Cha, and Hyuckjae Lee \\ School of Engineering, Information and Communications University (ICU) \\ 119 Munjiro, Yuseong-gu, Daejeon, 305-714, Korea \\ E-mail: \{myworldya, hschun, s1009, and hjlee\}@icu.ac.kr
}

\begin{abstract}
Adaptive modulation in OFDM systems is receiving remarkable attention as a scheme for improving spectral efficiency and error performance simultaneously. A major issue of the system is the necessity of channel information feedback that raises a problem of propagation delays. Linear channel prediction with drawback of biasing effect has been previously proposed to overcome propagation delays. In this paper, to avoid biasing effect, we propose channel prediction based on interpolation. Simulation results show that the proposed scheme has noticeable benefits of not only spectral efficiency but also error performance in OFDM systems using adaptive modulation.
\end{abstract}

\section{INTRODUCTION}

Orthogonal Frequency Division Multiplexing (OFDM) takes advantages that benefit from its robustness to multipath delay and its capability to support high data rate in wireless communication systems [1]. It has been adopted for various standards, such as digital audio broadcasting (DAB), terrestrial digital video broadcasting (DVB-T), and IEEE 802.11 wireless LAN. Furthermore, OFDM is considered as one of main candidate techniques for next-generation mobile communication systems. When the channel exhibits frequency selectivity, OFDM divides the bandwidth into narrowband subcarriers, and hence each subcarrier experiences individual frequency flat fading. Due to the distinct fading characteristics over subcarriers, it is very challengeable to apply an adaptive modulation scheme to OFDM systems to increase the spectral efficiency.

The adaptive modulation is an efficient way to optimizing the transmission rate in response to the channel condition, and it promises to achieve low error rate and high data throughput simultaneously [2]. To utilize its advantages, this scheme is also expected to be a dominant technique for the future generation mobile communication systems. For instance, it is recently discussed in lots of standards: packet data Code Division Multiple Access (CDMA), High Performance Radio LAN2 (HiperLAN2), and IEEE 802.11 system.

Adaptive modulation for OFDM systems employs different modulation types, depending on the channel side information (CSI) forwarded from the receiving end. In time-varying channel, modulation decisions may be made based on the outdated channel information caused by time delays during the feedback process. Most of previous studies on adaptive modulation have been reported without feedback propagation delays [3][5]. It was shown in [6] that wrong modulation decision owing to propagation delays results in significant performance degradation. In [7], linear channel prediction approach to handle the propagation delays was proposed. However, it has main drawback that long-range linear prediction with outdated channel information has inherent biasing effect, and hence the system suffers degradation of spectral efficiency.

In this paper, to avoid bias effect, we propose interpolationbased channel prediction on adaptive modulation in OFDM systems. In proposed algorithm, long-range prediction is made using the past data gathered at certain points. On the other hand, the existing prediction method utilizes the whole data. More specifically, some channel coefficients are predicted with sparsely sampled past values, and the others are estimated by interpolation approach. Unlike the linear prediction with biasing effect, the proposed scheme reduce the biasing effect significantly, and hence it provides the enhanced system performance. Computer simulation shows that the proposed prediction algorithm has noticeable benefits on spectral efficiency as well as error performance in OFDM systems with adaptive modulation.

This paper is organized as follows. Section II provides background of adaptive modulation in OFDM systems. In section III, the proposed channel prediction is described on detail, and the simulation results with some discussions are given in section IV. Finally, we make a conclusion in section $\mathrm{V}$.

\section{SYSTEM MODEL}

\section{A. $O F D M$}

A general OFDM system is briefly described here. Fig.1 shows a basic structure of OFDM systems. After symbol mapping, with Inverse Fast Fourier Transform (IFFT), data are subdivided to each subcarrier. To avoid ISI which would destroy the orthogonality of the subcarriers, cyclic prefix is inserted for a guard interval with length longer than the maximum delay spread. At the end of transmitter, cyclically extended OFDM frames are transmitted over the channel. At the receiver, the guard interval is removed, and take Fast Fourier Transform (FFT) with received data to transfer on frequency domain. Finally received data are decoded at demodulator. The overall OFDM system can be easily implemented using canonical channel modeling as shown in [8].

A noteworthy feature of OFDM is that it divides bandwidth into narrowband subcarriers, and frequency selectivity of the 


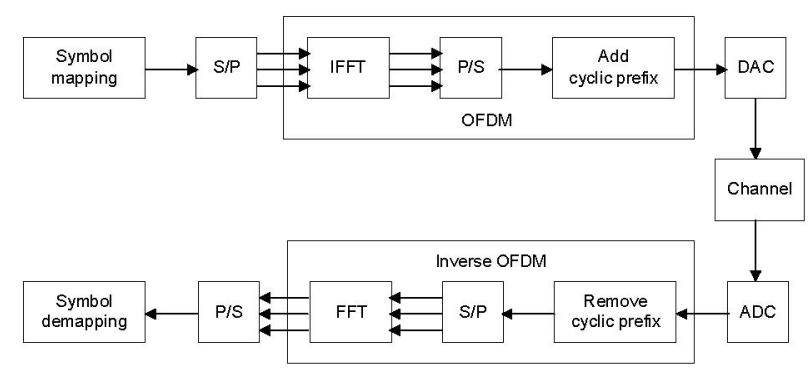

Fig. 1. Basic OFDM System Structure

channel provides distinct channel attenuations for each subcarriers. This property strongly motivates us to employ adaptive modulation in OFDM systems.

\section{B. Adaptive modulation}

Adaptive modulation is a way to improve the tradeoff between spectral efficiency and bit error rate. We are able to make such a optimization in Rayleigh fading channel by exploiting its fading dynamics. Unlike diversity concepts that average out the fading characteristics, adaptive modulation utilizes the fluctuation of the channel. In periods of high channel gains, instantaneous received SNR is high, and it naturally allows to use high order modulation. The situation of deep fading channel decreases received SNR, and limits the system to use low order modulation.

Specifically, in this paper, we use QPSK, 16-QAM, and 64QAM as modulation candidates for adaptive modulation. In order to decide proper switching levels from their BER performances, desired BER should be decided. We set our system's target BER performance to $10^{-3}$ which is required for reliable voice communications. With this object, our system will try to keep a BER lower than $10^{-3}$ with the most spectrally efficient modulation scheme. As a value for switching modulation, we decided to use SNR at the receiving end. In Rayleigh fading channel, the average of received signal power is same to that of transmitted signal power, thus it allows to consider the BER function of received SNR in an AWGN channel as the BER in Rayleigh channel in function of transmission SNR. Theoretical BER for M-QAM in AWGN with normalized transmission signal power are [9]:

$$
\frac{4}{\log _{2} M}\left(1-\frac{1}{\sqrt{M}}\right) \sum_{i=1}^{\sqrt{M} / 2} Q\left((2 i-1) \sqrt{\frac{3 E_{b}}{(M-1) N_{0}}}\right)
$$

Under $10 \mathrm{~dB}$ of SNR, no modulation gives BER below than $10^{-3}$. Between $10 \mathrm{~dB}$ and $17 \mathrm{~dB}$, QPSK only shows the desired BER performance. 16-QAM guarantees the required BER performance with the best spectral efficiency in SNR range of $17 \mathrm{~dB}$ to $23 \mathrm{~dB}$. In the SNR regimes over $23 \mathrm{~dB}, 64-\mathrm{QAM}$ can give the best spectral efficiency with the required $B E R$ performance. Therefore, we have the SNR boundary ranges for our adaptive modulation as Table I.
TABLE I

ADAPTIVE BOUNDARIES

\begin{tabular}{|c|c|}
\hline QPSK & SNR $<17 \mathrm{~dB}$ \\
\hline 16-QAM & $17 \mathrm{~dB} \leq \mathrm{SNR} \leq 23 \mathrm{~dB}$ \\
\hline 64-QAM & SNR $>23 \mathrm{~dB}$ \\
\hline
\end{tabular}

\section{Adaptive Modulation in OFDM}

To take benefits of both OFDM and adaptive modulation, adaptive OFDM system had been introduced [4]. Thanks to the characteristic of OFDM, the system is robust to multipath delay, and combining of adaptive modulation optimizes the transmission with maximizing the the throughput of the system.

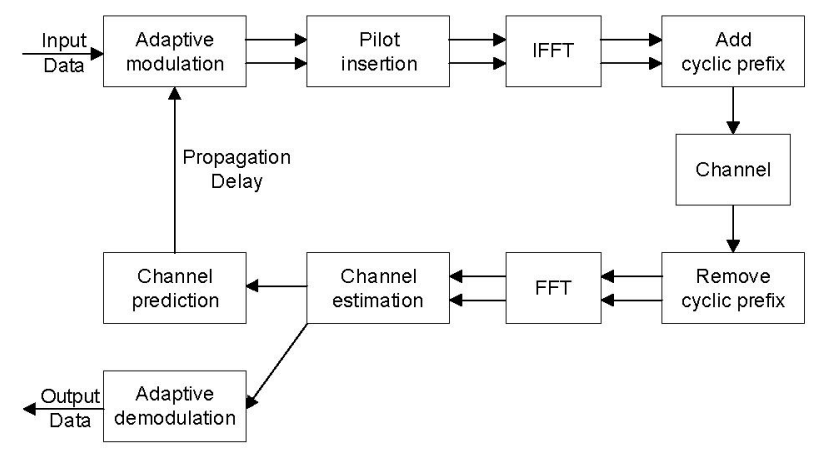

Fig. 2. Adaptive Modulation in OFDM System Block Diagram

Fig.2 represents block diagram of the adaptive OFDM system. It employs different modulation for each OFDM subcarrier according to the channel condition of the subcarrier. We utilize a pilot added channel estimation, and cyclic prefix is inserted to exclude multipath delays. After the receiving process and the channel estimation, a channel prediction is performed using the estimated channel information. Finally, received data are decoded, and the predicted channel values are transmitted back to the modulation switch at the transmitter.

\section{CHANNEL PREDICTION}

In adaptive modulation, the transmitter has to get present channel condition to employ proper modulation. Through the feedback path from the receiver to the transmitter, the estimated channel information are given back. The feedback channel is assumed error free, but this feedback causes time delays on the system, and it is called the propagation delays. In time-varying channel, because of this time consumption, the transmitter gets outdated channel information. Consequently, modulations are employed based on outdated information, and it causes serious performance degradations on adaptive modulation [6]. To exploit whole capability of adaptive modulation, we have to overcome this propagation delay, and for that purpose, usually channel prediction is used [7]. The basic principle of channel prediction is to use past channel information to predict future values. Fortunately, the correlative feature 
between values of Rayleigh fading channel makes possible to have such fundamentals. Using channel prediction, we could estimate what the future power level of the channel will be, hence the propagation delay could be got over.

\section{A. Long-range linear prediction}

The linear prediction estimates future value in the form of a linear combination between prediction coefficients and past values. With the correlative feature of the Rayleigh fading channel, prediction coefficients are determined using autocorrelations of past values. With the assumption for a stationary of the channel, the autocorrelation could be found as

$$
\phi_{j} \equiv\left\langle y_{i} y_{i+j}\right\rangle \approx \frac{1}{N-j} \sum_{i=1}^{N-j} y_{i} y_{i+j}
$$

where $y_{i}$ is the past value, and the linear prediction coefficient is given by [12]

$$
\sum_{j=1}^{M} \phi_{|j-k|} d_{j}=\phi_{k}, k=(1,2, \ldots, M)
$$

The estimated future value is expressed as a simple linear combination of prediction coefficients and past values

$$
c_{n}^{\prime}=\sum_{j=1}^{M} d_{j} c_{n-j}
$$

where $c_{n-j}$ is the past symbol, and $d_{j}$ is the channel prediction coefficient respectively. To predict multiple future symbols, it treats the latest predicted symbol as a actual present one. Using this methodology, it could predict as far as it desires. Fig. 3 shows a frame structure of the long-range linear channel prediction.

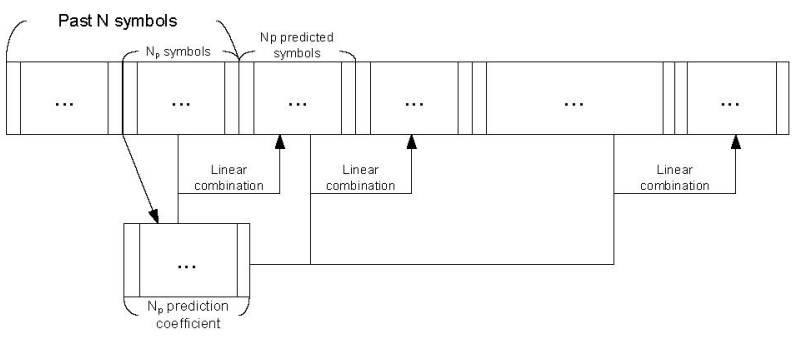

Fig. 3. Frame Structure of Long-range Linear Channel Prediction

However, to overcome the propagation delays, we need to predict long-range as far as the amount of delays. Longrange linear prediction generally uses identical prediction coefficients iteratively rather than to update because updating new coefficients needs a plenty of time. Ultimately, as we predict further with same coefficients, errors are accumulated, and the predicted values becomes lower than the actual ones. This is called biasing, and it causes significant lowering of throughput performance on the system [7].

\section{B. Proposed prediction}

To avoid biasing, we propose channel prediction based on interpolation. The block diagram of the proposed prediction scheme is represented in Fig.4.

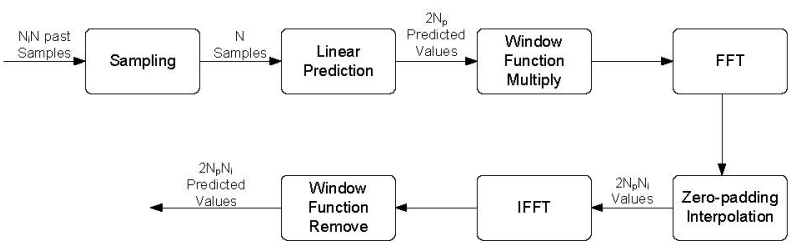

Fig. 4. Block Diagram of Proposed Channel Prediction

To begin with, the system has $N_{i} N$ past symbols. Among them take $\mathrm{N}$ uniformly distributed samples with $N_{i}$ sampling intervals. Using linear prediction, the predictor could get $2 N_{p}$ predicted samples where $N_{p}$ is the number of poles in linear prediction. To get whole $2 N_{i} N_{p}$ values, zero-padding interpolation in frequency domain is performed. Fig. 5 represents a frame structure of the proposed channel prediction.

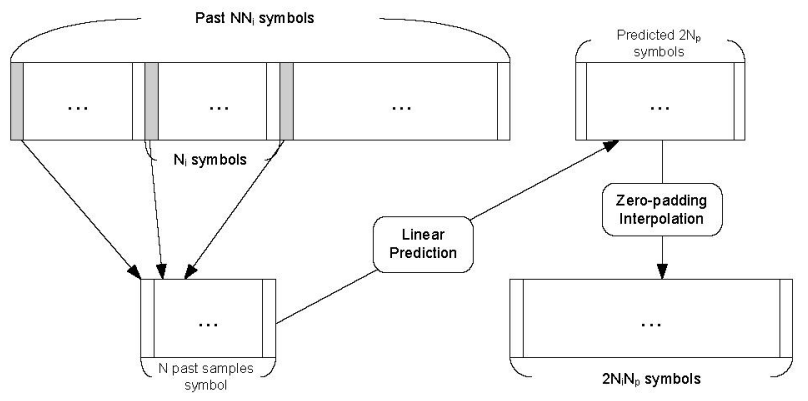

Fig. 5. Frame Structure of Proposed Channel Prediction

Since FFT and IFFT are used for interpolation, inserting window functions is effective in concentration of the signal spectrum. By using windowing, the spectrum power out of the calculation are decreased, so it could reduce the alias effect. In here, we use Hanning window function which represented as

$$
w_{l}=\frac{1}{2}\left[1-\cos \left(\frac{\pi l}{N_{p}}\right)\right]\left(l=0,1, \ldots, 2 N_{p}-1\right) .
$$

Window functions are multiplied with the predicted symbols.

$$
g_{1}(l)=g(l) w_{l},\left(l=0,1, \ldots, 2 N_{p}-1\right)
$$

Interpolator takes $2 N_{p}$ points FFT with the windowed symbols for zero-padding interpolation in frequency domain.

$$
G_{1}(n)=\sum_{l=0}^{2 N_{p}-1} g_{1}(l) \exp \left(-\frac{j \pi n l}{N_{p}}\right),\left(n=0,1, \ldots, 2 \mathrm{~N}_{p}-1\right)
$$

Zero-padding interpolation in frequency domain is represented like follows.

$$
G_{1}^{\prime}(n)=\left\{\begin{array}{l}
N_{i} G_{1}(n),\left[0 \leq n \leq N_{p}-1\right] \\
0,\left[\mathrm{~N}_{p}<n<N_{p}\left(2 N_{i}-1\right)-1\right] \\
N_{i} G_{1}\left(n-2 N_{p}\left(N_{i}-1\right)\right), \\
{\left[\mathrm{N}_{p}\left(2 N_{i}-1\right) \leq n<2 N_{p} N_{i}-1\right]}
\end{array}\right\}
$$


where $G_{1}(n)$ is predicted symbol in frequency domain. After zero-padding interpolation, we have $2 N_{p} N_{i}$ points in frequency domain. Fig. 6 shows the principle of zero-padding interpolation in frequency domain [10].

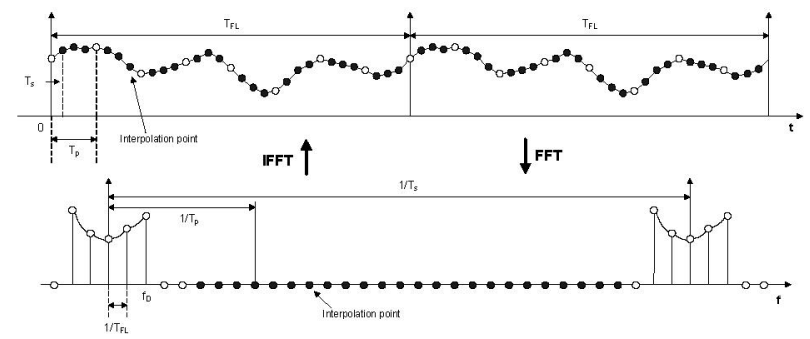

Fig. 6. Principle of Zero-padding Interpolation

After interpolation, by taking $2 N_{p} N_{i}$ points IFFT, interpolated symbols are converted to time domain.

$$
\begin{array}{r}
g_{1}^{\prime}(k)=\frac{1}{2 N N_{p}} \sum_{m=0}^{2 N N_{p}-1} G_{1}^{\prime}(m) \exp \left(-\frac{j \pi n l}{N N_{p}}\right), \\
\quad\left(k=0,1, \ldots, 2 \mathrm{NN}_{p}-1\right)
\end{array}
$$

After IFFT, de-windowing is performed by following function.

$$
w_{k}^{\prime}=\frac{1}{2}\left[1-\cos \left(\frac{\pi k}{2 N_{i} N_{p}}\right)\right]
$$

Finally, through the truncation and de-windowing, we could get $2 N_{p} N_{i}$ interpolated and predicted symbols.

$$
c^{\prime}(k)=\frac{g_{1}^{\prime}(k)}{w_{k}^{\prime}},\left(k=0,1, \ldots, 2 N_{i} N_{p}-1\right)
$$

\section{SIMULATION RESULTS}

To compare the performance of the proposed algorithm with linear channel prediction, we simulated adaptive modulation in OFDM with those channel prediction schemes. OFDM system with 64 subcarriers is used which is identical to IEEE 802.11 OFDM based WLAN standard. We utilize the comb-type pilot aided FFT channel estimation with interval of 15 symbols between each pilot [10], [11], and 1/4 of cyclic extended prefix is added to exclude multipath delays. Exponential channel with symbol duration $10 \mu \mathrm{s}$, rms delay of $5 \mu \mathrm{s}$, and $1 \mathrm{~Hz}$ of Doppler shift is used. In addition, the propagation delay of 256 symbols is assumed. In the linear channel prediction, we take 128 past symbols with 64 poles. In proposed scheme, 128 samples are taken among 512 past symbols, and same number of poles are employed.

Fig. 7 shows the BER performances of two schemes for adaptive modulation in OFDM systems. It is observed that the BER performances of two prediction schemes are analogous for overall SNR, but the proposed scheme has better performance at the SNR region around modulation decision boundaries. At high and low SNR regimes, the prediction error insensitive for employing modulation since they are far from the adaptive boundaries. However, in the SNR range of the adaptive boundaries, instantaneous modulation decision is

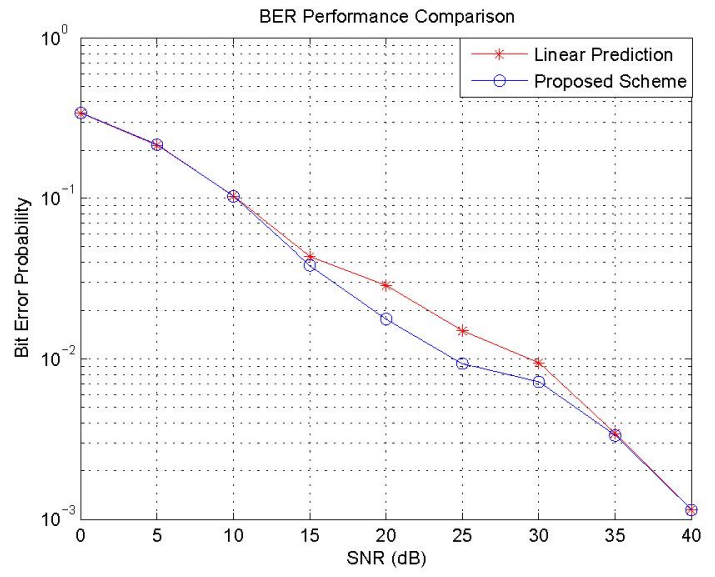

Fig. 7. BER Performance of Linear Channel Prediction and Proposed Scheme

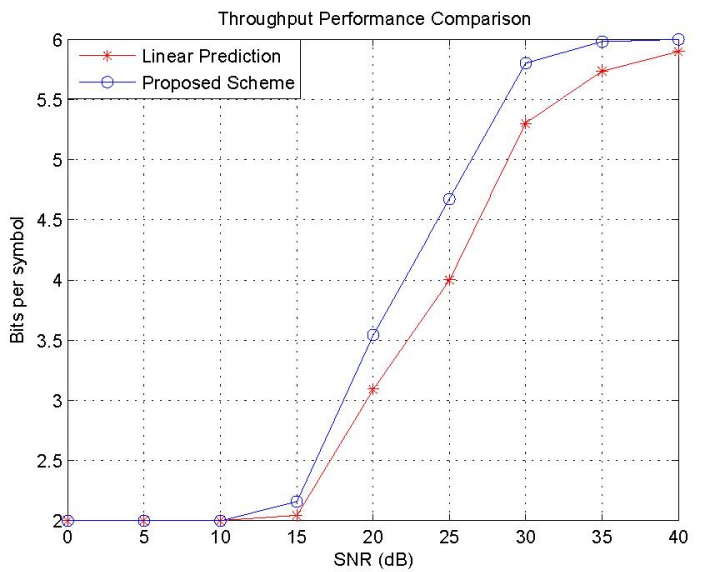

Fig. 8. Throughput Performance of Linear Channel Prediction and Proposed Scheme

relatively sensitive to the variation of the prediction error, thus the accuracy of channel prediction directly affects to the BER performance of the system. As a result, we could get up to $5 \mathrm{~dB}$ benefit of BER performance in the SNR region around adaptive boundaries

Comparison of the spectral efficiency is plotted in Fig.8. In the graph, throughput means the transmitted bits per symbol which increases as we can use higher order modulation. It can be observed that we could get considerably higher throughput performance with the proposed scheme. Throughput decrease in linear prediction is noticeable because the bias effect leading us to employ low order modulation. Meanwhile, the proposed scheme is much robust to biasing, thus throughput of the system considerably improved. Maximum performance gain is about $0.75 \mathrm{bits} / \mathrm{symbol}$ at the SNR around $25 \mathrm{~dB}$. In the sense of SNR gain, we could get up to $7 \mathrm{~dB}$ gain in high SNR regimes.

\section{CONCLUSIONS}

In this paper, we proposed interpolation based channel prediction for adaptive modulation in OFDM systems. The 
performance of adaptive modulation is very sensitive to the accuracy of the channel prediction. As demonstrated in simulation results, more correct prediction is possible with the proposed scheme. Moreover, it gives the robustness to biasing effects, compared to the existing long-range linear prediction. Therefore, the proposed channel prediction can considerably improve not only the system throughput but also the bit error rate in OFDM systems using adaptive modulation technique.

\section{ACKNOWLEDGEMENT}

This research was supported jointly by Samsung-ICU 4G Research Center and by the MIC, Korea, under the ITRC support program supervised by the IITA.

\section{REFERENCES}

[1] I. Kalet, "The Multitone Channel," IEEE Trans. on communications, vol.37, pp.119-124, February 1989.

[2] J.M. Torrence and L. Hanzo, "Upper Bound Performance of Adaptive Modulation in a Slow Rayleigh Fading Channel," IEEE Electronics Letters, vol.32, p. 718, April 1996.

[3] Y.T. Hwang, et al., "Block-wise Adaptive Modulation for OFDM WLAN Systems,'ISCAS'05, vol.6, pp.6098-6101, May 2005.

[4] A. Czylwik, "Adaptive OFDM for Wideband Radio Channels," GLOBECOM'96, vol.1, pp.713-718, November 1996.

[5] M. R. Souryal and R. L. Pickholtz, "Adaptive Modulation with Imperfect Channel Information in OFDM," ICC'O1, vol.6, pp.1861-1865, June 2001.

[6] D. L. Goeckel, "Adaptive Coding for time-varying channels using outdated fading estimateds,'IEEE Trans. on communications, vol.47, pp.844855, February 1989.

[7] R. Chan, "Channel Prediction for Adaptive Modulation in Wireless Communications," Master Thesis, Blacksburg, Virginia, July 2003.

[8] M.Guillaud and D. T.M. Slock, "Channel Modeling and Associated Intercarrier Interference Equalization for OFDM Systems with High Doppler Spread," ICASSP '03, vol.4, pp.237-240, April 2003.

[9] L. Jianhua and K. B. Letaief, "M-PSK and M-QAM BER Computation Using Signal-Space Concepts,'IEEE Trans. on communications, vol.47, February 1999.

[10] E. Okamoto, et al., "Rayleigh Fading Compensation for 16 QAM Using FFT," PIMRC'96, vol.3, pp.1079-1082, October 1996.

[11] S. Coleri, et al., "Channel Estimation Techniques Based on Pilot Arrangement in OFDM Systems," IEEE Trans. on broadcasting, vol.48, September 2002.

[12] W. H. Press, et al., Numerical Recipes in C, Press Syndicate for the University of Cambridge, 1997. 